\begin{tabular}{|ll|}
\hline Received & $: 10$ Agustus 2020 \\
Revised & $: 13$ Desember 2020 \\
Accepted & $: 14$ Desember 2020 \\
Online & $: 15$ Desember 2020 \\
Published & $: 18$ Desember 2020 \\
\hline
\end{tabular}

\title{
Understanding Basics Holography: Review Principle and Recording Technique of Holography
}

\author{
Irsad Tio Majid $^{1, \text { a }}$ \\ ${ }^{1}$ Departement of Physics, State University of Jakarta \\ Rawamangun, Jakarta, Indonesia, 13220 \\ ${ }^{a)}$ IrsadTioMajid_1306618028@mhs.unj.ac.id
}

\begin{abstract}
In this article, we review the basics of holography. Start to knowing the description of holography, mathematical processing, etc. That also include the principle physics phenomenon in Optical field, the trajectory (Technique) hologram, and the difference about optical holography, and digital holography. Holography Technology had been applied to much various cases.
\end{abstract}

\section{INTRODUCTION}

The term holography is well known as, "depth perception from two-dimensional surface or film". Its becomes popular because the image from a good hologram resembles the original object itself. But in scientific terms, holography can't just to be described as a display technique with an extra dimension. Even the discovery of this technique did not originate from any optical recording or display related research (Johnston, 2006). Holography was being technology of optics that records and reconstructs wave-fronts of light and have potential development for use in next- generation imaging technology with various applications such as optical data storage and three- dimensional holographic imaging (Geng, 2013). This technique was invented by Dennis Gabor in 1947 when he was working on to improve his electron microscope (Gabor, 1951) (Gabor, 1948). Holography is popularly known as a display technique because we have good coherent sources only in the visible spectrum. The principles of holography suggest that it is much more capable than that. To realize and appreciate all the potentials of this technique, it is necessary to understand the science behind it.

Holography became the art of records the complete data information object and reproducing them in a later time. These records of both phase information and amplitude contained within emanating wave from the object. Optically, the hologram could be defined as diffraction screen when suitably illuminated, diffracts light in the desired manner. These hologram could be made to resemble the light than would otherwise emanate from an object when illuminated. Then, if we see the illuminated screen, like we see the real itself. The pattern of diffraction inscribed in the hologram was responsible for the process. The art of inscribing and generating pattern on the holographic screen that missing to be holographic recording (B J \& P K, 2013).

Dennis Gabor's solutions had to wait for 30 years until the invention of the laser, for the first hologram to be made. The earliest and the popular method of recording the pattern on the hologram is by using 
highly coherent light sources (laser) and high-resolution photosensitive recording materials (Hariharan, 2002). The object was illuminated with laser, and the light that bounces back from the object reaches the recording medium. Another beam from the same laser directly reaches the recording medium. Both these beams interfere in the recording medium to produce the hologram. Different recording setups were proposed each having their own significances. Even holograms that could be reconstructed with an incoherent light like sun light were made using this procedure. The perception of a good quality hologram was breathtaking. These method in records and reconstruct was generally termed as conventional holography or optical holography.

Optical holography demands the presence of the real object during the recording process. The solution came from Lohman and Paris (Lohman \& Paris, 1967) in the form of computer-generated holograms. In this process, the optical holographic recording setup was simulated to generate the pattern that the real object would otherwise produce. Now, the generated pattern is transferred to a transparency film and reconstructed using a laser. The pattern was very attractive, because we have virtually created an object that never existed. This also provided other conveniences like not demanding a costly optical recording setup or vibration isolation arrangement. Scalar diffraction theories (Born \& Wolf, 1999) were used to simulate all the process as (diffraction, wave propagation and interference) involved in the optical recording setup. This method of recording and reconstruction is popularly known as Digital holography.

\section{PRINCIPLE OF HOLOGRAPHY}

Light is some of electromagnetic wave in nature and became standard on theory of holography which entirely revolves around electromagnetic wave propagation (Born \& Wolf, 1999). Some of holographic records and reconstructed setup being considered for explaining the foundations of theoretical holography. The principle of holography also consists the records of hologram by interference between reference wave and object wave followed by the propagation and diffraction of another reference wave that resulting in the formation of the holographic image. There were being illustrated with two elementary holograms: holography of point sources and plane waves. Holography could be realized through an optical processes and large range of materials (Kim, 2011)

A hologram requires two coherent beams and the holographic plate records their interference pattern. In practice, both beams derive from the same source, one serves as a direct reference beam the other is the wave-front scattered from the object (Pain, 2005). Figure 1 shows one possible arrangement where a partly silvered beam splitter passes the direct reference beam and reflects light on to the object which scatters it on to the photographic plate at y-plane.

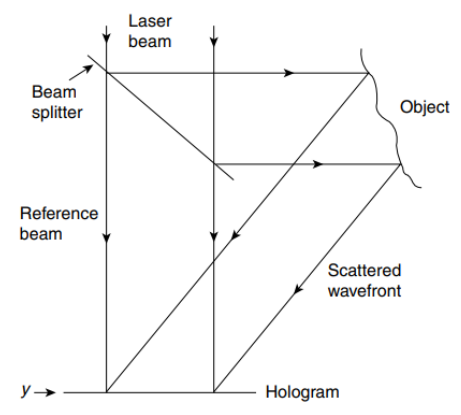

FIGURE 1. The hologram records the interference between two parts of the same laser beam (Pain, 2005). 
From figure 1, we can conclude the part to be done in one dimensional hologram. But, if we want to infinite the basic, we must past over the one-dimensional hologram and focus to trying in threedimensional hologram. The principle of holography must consist the light source, recorded object, and recording object. Light with sufficient coherence length was split to two partial-wave with using beam splitter. The first wave illuminate the object and the other was reference wave. Both of wave interfere to create a standing wave pattern. These interference pattern known as hologram we've been reconstructed. To mention the recording and reconstruction process, there was some object that can be wave displacement.

$$
U(x, y)=A_{0}(x, y) e^{i \phi(x, y)}
$$

with the amplitude and phase,

Hence, the intensity became,

$$
R_{r}(x, y)=A_{r}(x, y) e^{i \psi(x, y)}
$$

$$
\begin{gathered}
I(x, y)=\left[U(x, y)+R_{r}(x, y)\right]^{2} \\
I(x, y)=\left[A_{0}(x, y)\right]^{2}+\left[A_{0}(x, y)\right]^{2}+2 A_{0}(x, y) A_{r}(x, y) \cos (\psi(x, y)-\phi(x, y))
\end{gathered}
$$

At the first place, exposure must be correctly chosen at the value of optimal exposure. Secondly, the value of reference beam intensity $A_{0}^{2}$ must be chosen to produce the correct transmitted amplitude on the vertical axis in figure 2. This value of amplitude transmittance is at the center of the linear range. Finally, the modulation of $A_{0}^{2}$ by the scattered intensity $A_{r}(x, y)^{2}$ in figure 2 must be small enough for the transmission of the modulated signal to remain within the linear range of the characteristic curve (Pain, 2005). Excursions outside the range will introduce non-linear distortions by generating extra Fourier frequency components.

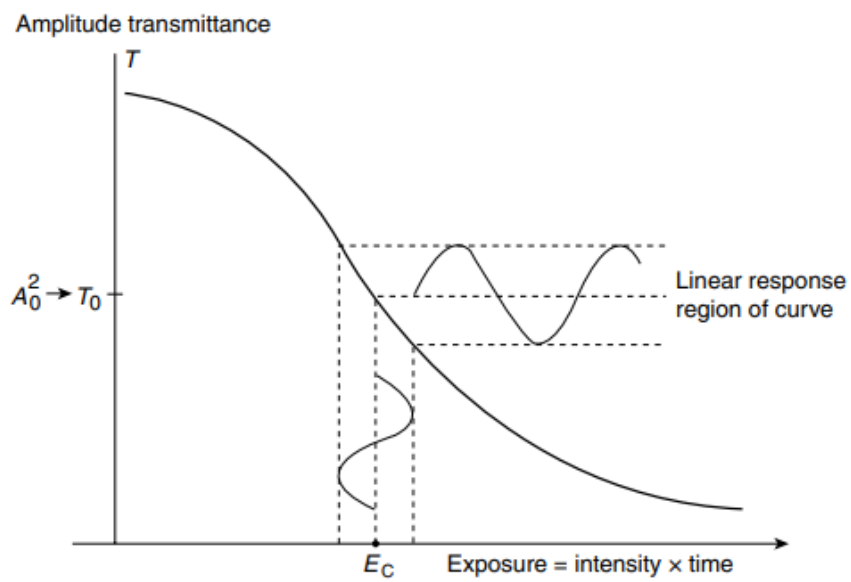

FIGURE 2. Characteristic curve of a real holographic emulsion (transmittance versus exposure). Only the central linear section of the curve is used. The transmittance was chosen with the critical exposure to produce the central point on the linear part of the curve. Information from the scattered wave-front must keep the modulations within the linear range for faithful reproduction free from distortion (Pain, 2005)

Apart from these, there's an awfully between the important and reflection. Since the important image is made by the conjugate object wave $u(x, y)$, it's the curious property that its depth is inverted. Corresponding points of the reflection and real image square measure situated at equal distances from the 
photographed plane, however at opposite sides of it like figure three. The background and foreground of the important image square measure so changed. the important image seems inverted. This image is termed "pseudoscopic" contrary to the conventional image that is termed "orthoscopic" (B J \& P K, 2013). Also, optics studies were partly derived from 2 technology. it had been Optical optics (OH) and Digital optics (DH).

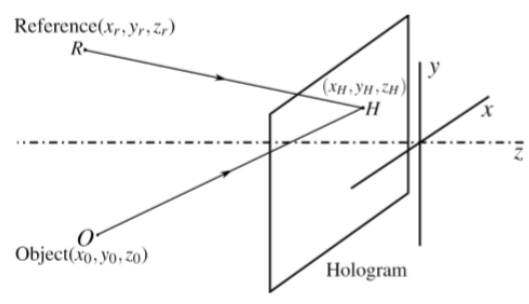

a)

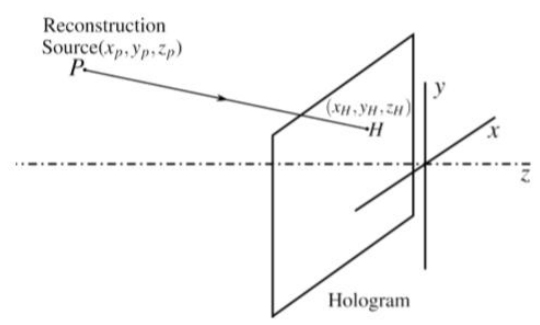

b)

FIGURE 3. Example for the processing hologram. a) Hologram recording, and b) image reconstruction (Hariharan, 1996)

\section{OPTICAL HOLOGRAPHY (OH)}

The method of holography in recording and reconstruction was done using laser light on a holographic plate called as $\mathrm{OH}$. The popular method to making holograms which demands the presence of real object and highly stable vibration free recording environments. The reconstruction is done either with laser or white light (Jeong, 1967). The best quality holograms that very closely resemble the object were made using this procedure. No digital electronic devices were used for recording or reconstruction because its $\mathrm{OH}$. For the principle of $\mathrm{OH}$, there were depends on the using method. But the basic had been explained at the previous section. Hence, we explain the several methodologies used in $\mathrm{OH}$.

\section{OH Method}

There are a lot of methods available for recording and reconstructing holograms optically. Each method has its own merits and demerits. This section discusses briefly each method and its significances. This paper may need to be updated after announcement of new $\mathrm{OH}$ methods. Also, the full version of description, we refer to read (Ackermann \& Eichler, 2007) and (Hariharan, 2002; Hariharan, 1996).

\section{Inline Hologram}

Inline Hologram was the first technology in holography. The light was going to an incident on the photographic plate then contains two components. The first is the directly transmitted wave, which is a plane wave whose amplitude and phase doesn't have variety across the photographic plate. The second became a weak scattered wave that emanated from the main object. Both these waves superimpose on the photographic plate giving rise to the fringe pattern which is the hologram (Gabor, 1948). 


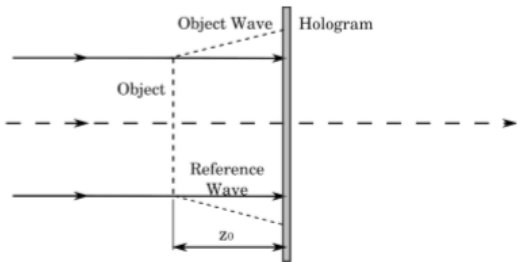

a)

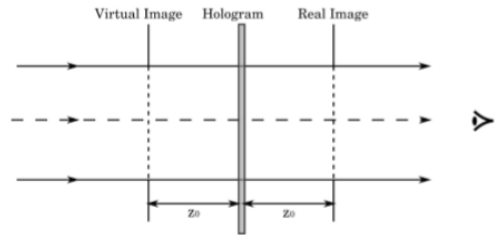

b)

FIGURE 4. processing In-lane Hologram. a) Hologram recording, and b) image reconstruction (Hariharan, 1996)

If the object had been chosen as an axial point $\mathrm{O}$ emitting spherical wave, the result of hologram for a plane reference wave can be defined a Fresnel zone lens. During reconstruction, the hologram is illuminated with a plane reference wave as shown in Figure 4(b). A virtual image point is formed at the original object position and additionally, a real image point appears at the same distance to the right of the hologram. The phenomena also hold for extended objects that would be divided into single points.

\section{Off-Axis Hologram}

The recording arrangement for off -axis holography is shown in figure 5. The reference beam became a collimated beam of uniform intensity, derived from the same source to illuminate the object. By tilting the reference wave (or shifting the object) the three diff raction orders, namely the image, the conjugated image, and the illumination wave, are spatially separated.

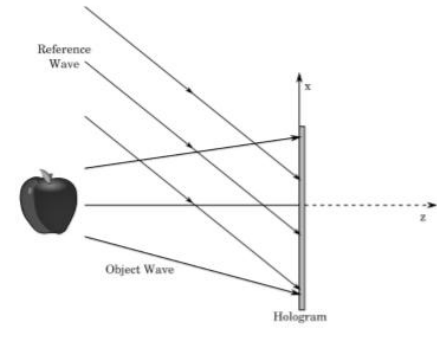

a)

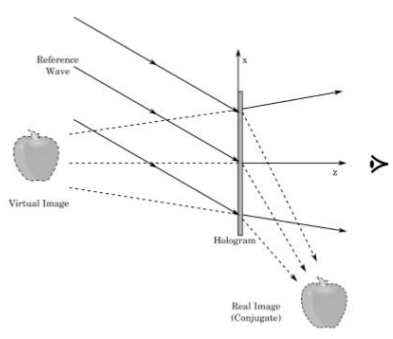

b)

FIGURE 5. processing off-axis Hologram. a) Hologram recording, and b) image reconstruction (Hariharan, 1996)

This is the most popular and widely used method for recording holograms optically. But on the contrary this is the least used method by digital holographers. This is due to the fact that the fringe density increases with angle between the reference and object.

\section{Fourier Hologram}

The optical setup for recording a Fourier hologram is shown in figure 6. The plane object was placed in the first focal plane of the lens. The reference wave emerges from the source of light point in the same plane. The photographic layer is placed in the back focal plane of the lens during recording (Vander, 1964). The reconstruction is done by illuminating the hologram with an axially parallel plane wave as 
shown in Figure 6(b). The hologram was placed propagate in the first focal plane of a similar second lens. The primary and the conjugated images could appear in the second focal plane-symmetric to the optical axis. The un-diff racted reference waveforms an axial light spot that representing the zeroth diff raction order. It can be shown the reconstructed image remains stationary when the hologram being shifted in its plane.

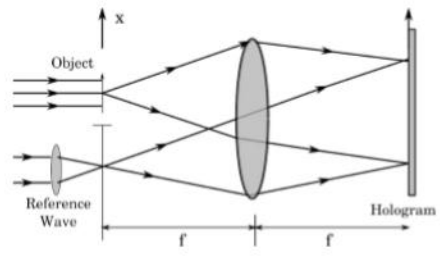

a)

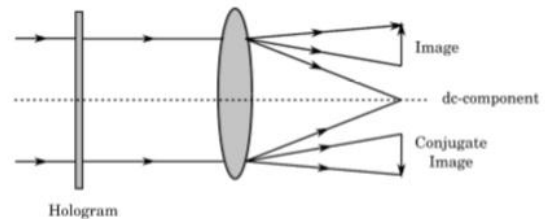

b)

FIGURE 6. processing Fourier Hologram. a) Hologram recording, and b) image reconstruction (Hariharan, 1996)

Fourier holograms had been the useful property for who reconstructed image that does not move when the hologram was translated in its own plane. because a shift function in the spatial domain only results in a form of Fourier transform being multiplied by a phase factor which has no eff ect on the intensity distribution (Hariharan, 1996). This setup is most liked by digital holographers because the simulation is very easy which only requires an FFT calculation. The Fourier holograms are limited only to plane objects and 3D perception is not possible (B J \& P K, 2013).

\section{Fraunhofer Hologram}

Different from Fourier Hologram, a hologram of this type is especially used for the measurement and investigation in aerosols (Trolinger, 1975). A Work scheme for Fraunhofer Holograms shown in figure 7. The object with radius $z$ so small that effected to a diff raction pattern will appear in the far field. The condition for any distance between the object and hologram is $z_{0} \ll \frac{z^{2}}{\lambda}$.

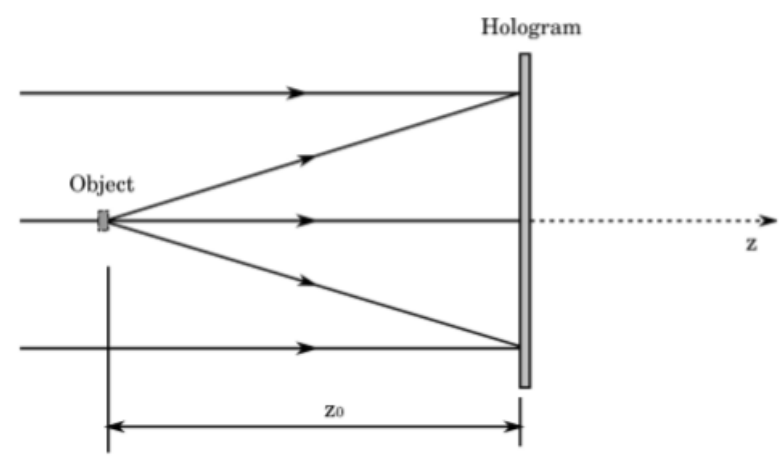

FIGURE 7. Optical System for Fraunhofer Hologram (Hariharan, 1996) 


\section{Rainbow Hologram}

Rainbow holograms can be reconstructed in transmission using white light (Benton, 1977; Ackermann \& Eichler, 2007) Depending on the viewing direction the reconstructed image appears in a different spectrum, exhibiting the whole light spectrum. The recording and reconstruction setup shown in figure 8. The technique to records rainbow hologram consists of two steps. In the first step, an off -axis hologram is created in the usual manner (Figure 8(a)). In the second step, a photosensitive layer can be positioned inside the real image and a second hologram $\mathrm{H} 2$ is created as shown in Figure 8(b). By the processing, the information of the pseudoscopic image was being recorded.

To reconstruct the rainbow hologram images, they were rotated by half-full clock to create an orthoscopic image from the pseudoscopic one. The image is reconstructed using monochromatic light as shown in Figure 8(c). When using the white light for reconstruction, the image of the horizontal slit appears under a diff erent diff raction angle, see Figure 8(d). For each spectral line, there exists a diff erent viewing slit.

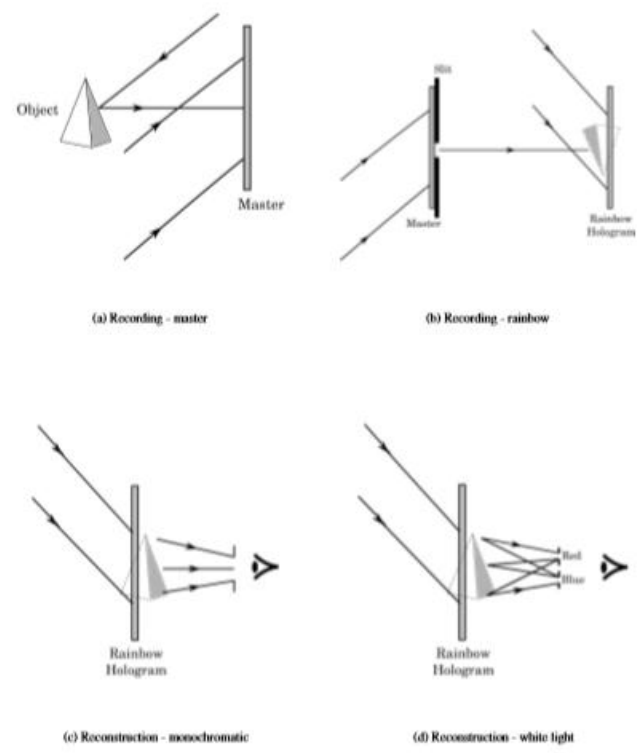

FIGURE 8. Optical System for Fraunhofer Hologram (Ackermann \& Eichler, 2007)

\section{Cylindrical Hologram}

A drawback experienced by all the holographic methods discussed so far is the limited angle over which they can be viewed. This is because they were all made only on plane surfaces. The cylindrical holography is supposed to make the complete geometry of the object viewable. It can be recorded by using a cylindrical film surrounding the object. Figure 9 shows the setup for a single-beam transmission hologram, proposed by (Jeong, 1967). 


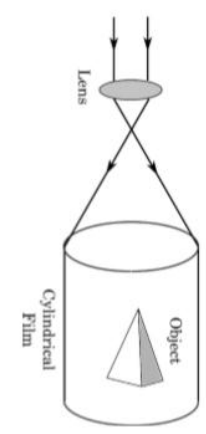

FIGURE 9. Optical system recording in cylindrical hologram (Ackermann \& Eichler, 2007)

The object was placed on the glass cylinder center which has a strip of photographic film taped to the inner surface with the emulsion side facing inwards (Hariharan, 2002). The expanded laser beam being some incident on the object from above. The central portion of the expanded laser beam illuminates the object gets scattered and reaches the cylindrical hologram surface, which constitutes the object beam. While the outer object can fall directly on the film being constituted the reference beam. The object and reference beams interfere in the cylindrical hologram surface to generate the hologram.

To view the reconstructed image, the processed film was replaced to the original position and illuminated with the same object as the laser beam. When illuminating the object, all perspectives of the object are displayed when walking around the hologram. Multiple holograms are also often reconstructed using the 360 Geometry degree. With illumination from above, all recordings of the multiple holograms can be reconstructed simultaneously and the recorded changing images can be observed when walking around the complete circle. Hence this method is also called one full clock rotation holography.

\section{OH Application}

$\mathrm{OH}$ have several application in generous human life. It's work to help some of human work from some scale and their needed. Some application of $\mathrm{OH}$ could be an security and authentication. Security and authentication is most common area for holography and diffractive optics applications. The demand on security being dictated by steady to growing of piracy and counterfeiting (Andrelevicius, 2011). There so many advantages to bring oh technology, like the most $\mathrm{OH}$ are designed to make positive impression, easy to recognizable by inexperienced user, complicated manufacturing of $\mathrm{OH}$, and inexpensive. Second application was being to measurement, particle analysis, and data storage.

\section{DIGITAL HOLOGRAPHY (DH)}

We know that during the holographic recording or reconstruction process, if the hologram is in digital form, then the technique is called as Digital holography. It is usually done by simulating the optical holographic recording or reconstruction setup on a computer. The schematic of this process is shown in figure 10. 


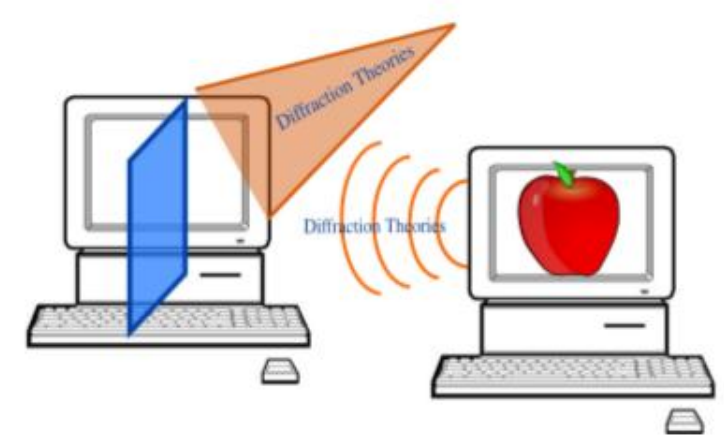

(a) DH - Computer generated

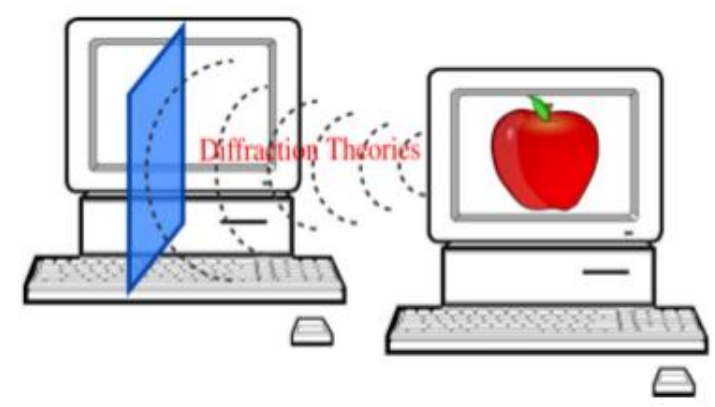

(b) DH - Computer reconstructed

FIGURE 10. Schematic of Digital Holography (B J \& P K, 2013)

Figure 10(a) represents the process where the object to be recorded is modeled in the computer. Then, the wave propagation from object to the hologram plane is simulated. The same simulation is done for the reference, and the interference between the object and reference is calculated at the hologram plane. Hence, the required hologram is generated. This generated hologram is in digital format and can be transferred to a photographic film using image setter or other methods. Then the photographic film can be reconstructed using optical methods. Thus hologram of an object that does not exist or cannot be optically recorded, can be produced using this method. Figure 10(b) shows the process in which the hologram is recorded optically, but a CCD is used for recording instead of holographic plate. Thus the recorded hologram is in digital format. The hologram is fed into a computer and reconstructed on the computer. The wave propagation is simulated from hologram plane to object plane. The object that was recorded can be viewed in the computer screen. Simulating wave propagation from object to hologram plane or vice versa, occurs in both the process and is the most important step in digital holography. This is basically a signal processing problem.

In this article, we just introduced the DH on Computer-generated hologram (CGH). About Computerreconstructed hologram (CRH), you can view (Ozcan \& Bayraktar, 2009) for the methods, and for mathematical principle in (Acharya \& Upadhyay, 2015). On Computer-generated hologram, we compared the theory of DH in (Poon, 2006) in these article. 


\section{CGH Principle}

As we know, Holography technology require the wave equation to understand it. Also, the basic field to learn the foundation of $\mathrm{CGH}$ were diffraction and interference. Approaches to solving diff raction problems can be investigated under four categories. From rather simple to more complicated categories, these categories are ray optics, wave optics, electromagnetic, and quantum optics. Ray optics describes the propagation of light by using geometrical rules and rays (Saleh \& Teich, 1991). In wave optics, the propagation of light is described by a scalar wave function which is a solution of the wave equation. Other signal processing approaches have also been extensively employed in problems related to wave optics. Another problem part of fundamental laws nature was the discretization of signals associated with propagating optical waves.

The propagation of electromagnetic field is defined by the wave equation. Analytical solution to wave equation describes the wavefield due to a propagating wave front anywhere in space. But in digital holography, the object has arbitrary shape and size and hence analytical solutions to the wave equation is Impossible to be done. Hence, numerical solution to the wave equation is sought to calculate the wavefield in the hologram plane or reconstruction plane. Wave equation is a vector differential equation and numerically solving it is very time consuming. Moreover, sampling errors and discretization errors creep in when the distance of propagation increases, aff ecting the results very badly. To overcome these issues, approximations have been induced into the equation based on the problem in hand. The approximated equations are integral equations derived from the Helmholtz differential equation using a suitable Greens function. These integral equations are scalar in nature and hence are also called as scalar diff raction formulas. These approximated solutions make calculation much easier and faster, but at the same time give satisfying results in holography.

We determined the theory from the four-Maxwell equation to give the single scalar wave equation. If the medium of propagation is linear, isotropic, homogeneous, and nondispersive, all components of electric and magnetic field behave identically and their behavior is fully described by a single scalar wave equation.

$$
\nabla^{2} V(x, y, z, t)-\frac{n^{2}}{c^{2}} \frac{\partial^{2} V(x, y, z, t)}{\partial t^{2}}=0
$$

For a monochromatic wave, scalar field written explicitly,

$$
\begin{aligned}
& V(x, y, z, t)=U(x, y, z) e^{-i 2 \pi v t} \\
& U(x, y, z)=U(P)
\end{aligned}
$$

If this scalar field represents a propagating optical field, it will became Helmholtz Equation. It can be very well stated that the complex amplitude of any monochromatic optical disturbance propagating in vacuum $(\mathrm{n}=1)$ or in a homogeneous dielectric medium $(\mathrm{n}>1)$. The next phenomena was Diffraction. Diff raction is a phenomenon of considerable importance in the fields of physics and engineering whenever wave propagation is involved (B J \& P K, 2013). We explain the theory in easy way to figure 11. 


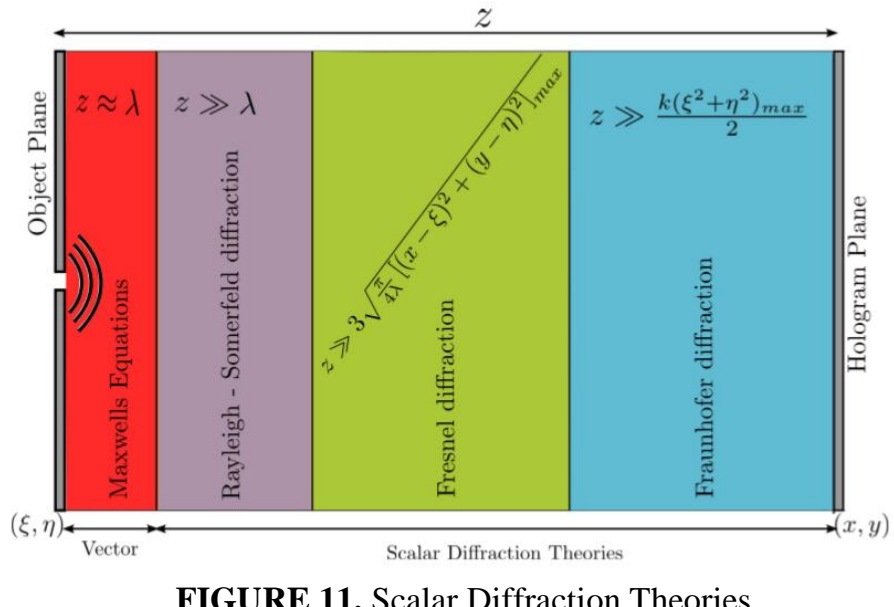

Light is also electromagnetic in nature and hence the disturbance (complex amplitude) of a propagating light field can be determined anywhere in space and at any time by the Maxwell's equations. Numerically evaluating the complex amplitude using Maxwell's equation is very difficult due to its vectorial nature and also the discretization errors that creep in. But when the medium of propagation becomes, linear, isotropic, homogeneous, and nondispersive the Maxwell's equations can be greatly simplified to get rid of the vectorial nature. This gives rise to the scalar wave equation as seen from Equation (4). Experiments in digital holography satisfy these requirements and hence scalar diff raction theories are the mostly used ones to simulate wave propagation. The scalar wave equation which is a diff erential equation can also be expressed as an integral equation based on a particular Green's function. It also turns out that the Huygens-Fresnel postulate on diff raction can be mathematically expressed using this integral equation as seen from Equation (6)

$$
U(P)=-\frac{i A}{\lambda} \int_{A} \frac{e^{i k(r+s)}}{r s}[\cos (n, r)] d S
$$

These non-vectorial integral equations constitute the Scalar Diff raction theory. These equations can be further approximated based on the propagation distance which results in the Fresnel and Fraunhofer diff raction theories. These approximated theories reduce the computation complexity greatly and fit well into most practical situations. Hence, the Fresnel and Fraunhofer diff raction theories are the mostly used ones in digital holography. But they can be used with Fast Fourier Transform (FFT) only when the object surface and hologram surface are parallel to each other and perpendicular to the optical axis. There can be situations where the hologram surface is tilted or curved to the object surface.

All the scalar diff raction theories explained in this chapter define wave propagation in real space except the angular spectrum formula Equation (7)

$$
U(x, y, 0)=\iint_{-\infty}^{\infty} A\left(f_{x}, f_{y}, 0\right) e^{i z \sqrt{k^{2}-4 \pi^{2}\left(f_{x}^{2}+f_{y}^{2}\right)}} e^{i 2 \pi\left(f_{x} x+f_{y} y\right)} d f_{x} d f_{y}
$$

Which defines propagation in spectral domain. This formula can also be evaluated by FFT, only if the object and hologram surface are plane and parallel to each other i.e., are shift-invariant. For the recommended FFT three dimensional in hologram, we recommended to read (Poon, 2006). 


\section{DH Generated Method}

Instead of optical recording, the hologram associated with the wavefront representing the object is generated by employing diff erent computational techniques and numerical approaches by mathematically simulating the optical wave propagation. An ideal CGH could be achieve complex light modulation at a high diff raction efficiency and precise reconstruction on the target image. The CGH's outperform conventional refractive and diff ractive components as a consequence of their ability to create any desired wavefront and thus to modify the input wavefront with much better flexibility (Flury et al. 2005). For this reason, CGHs find a wide range of application as display elements, optical interconnects, aberration compensators in optical testing, spatial filters for optical signal processing and computing, beam manipulators, and array generators etc.

CGH's can be considered as thin optical elements with a complex amplitude transmittance. However, in many cases, they are phase only elements (Davis et al. 1999). There are diff erent classification of CGHs depending on the complex amplitude representation on the recording media (binary, phase, amplitude and combined phase-amplitude media), and the encoding method (Yaroslavsky 2003). The algorithm to form a CGH is chosen according to the desired image characteristics and the associated computational complexity. Analytical approaches such as phase-detour method, kinoform method, double or multiple phase methods, explicit spatial carrier methods, 2-D simplex representation, representation by orthogonal and bi-orthogonal components, coding by symmetrization, etc., can be used for computing digital holograms (Yaroslavsky 2003). There are cell-oriented and point-oriented methods. In celloriented CGH's the hologram plane is divided into small resolution elements. The number of resolution cells needed depends on the complexity of the wavefront that is to be produced. Iterative approaches such as iterative Fourier transform algorithm (Wyrowsky and Bryngdahl 1988), direct binary search (Seldowitz et al. 1987), simulated annealing (Kirk and Hall 1997) have been proposed and used. These methods are computationally demanding.

\section{DH Reconstruction Method}

We could not afford the principle of CRH. Authors need to explain the reconstruction method to give an imagination about DH Method. There was several methods of Reconstruction of DH.

\section{Holographic Display}

Holograms are stored as digital images in the computer which can be streamed into any display devices one after the other. Hence, a dynamic display device is required to realize the video capabilities of digital holography. Candidate technologies for holographic display units include dynamically writable/erasable chemical films (Beev, et al., 2006) or on electronically controllable arrays of pixels that can alter the phase and amplitude of light passing through them, called spatial light modulators (SLMs) (Amako, et al., 1993). Specific forms, like deflectable mirror array devices (DMADs) are also among potential technologies that can be adapted for 3-D display, which can also be considered as special forms of SLMs (B J \& P K, 2013). Currently, dynamic chemical film technology is not mature enough for acceptable performance. Unfortunately, the size, quality and geometries of SLMs are insufficient for acceptable quality $3-\mathrm{D}$ displays. 


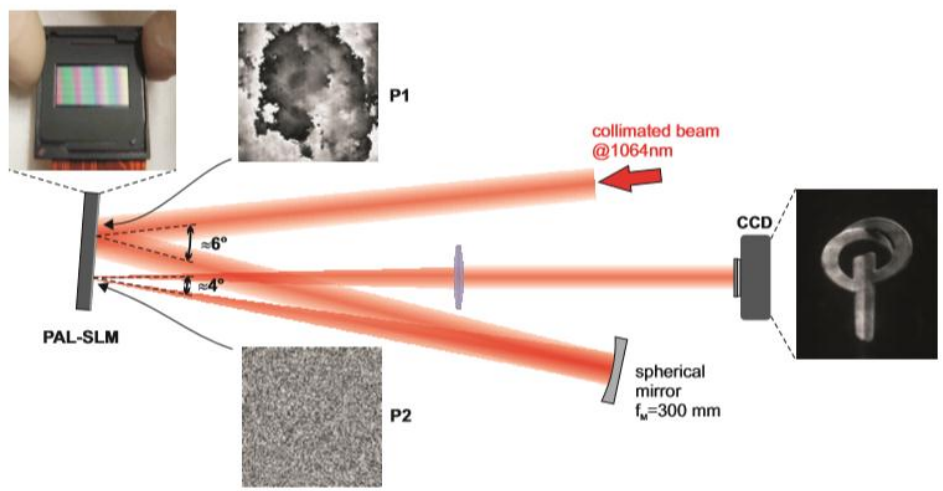

FIGURE 13. Example of Sketch from Holographic Display (Jesacher, et al., 2008)

Ability to steer light from each point of a display device to arbitrary directions provides solutions to the dynamic display problem. Speckle noise in case of coherent illumination is another disadvantage, and there are proposed techniques to cope with this problem (Iwai and Asakura 1996).

\section{Integral Imaging}

Integral photography has been revitalized after the progress in active pickup devices and microlens manufacturing processes (Okano, et al., 1997). It relies on a capture device based on a microlens array to encode a true 3-D optical model of the object as a planar intensity distribution which can then be reconstructed by reversing the direction of incident optical rays. Analysis of integral imaging devices can be carried out both by ray and diff ractive optics. Improvements in related computational procedures and the incorporation of novel techniques like moving lens arrays solved many problems in integral imaging. The maximum information capacity of integral imaging can be increased by using Karhunen-Loeve transform for image compression. Holograms can be computed from captured images during integral photography. The application of these can be seen in figure 13 .

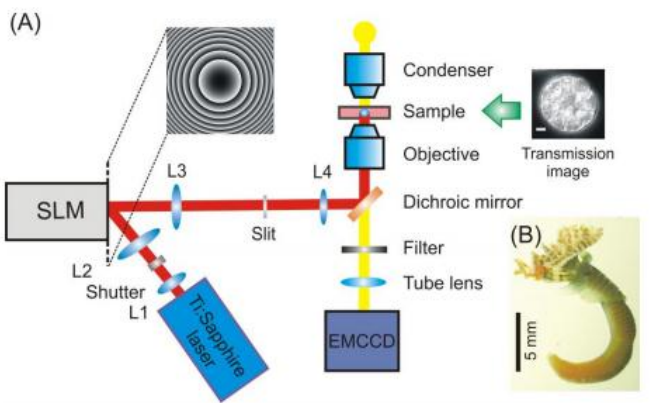

a)
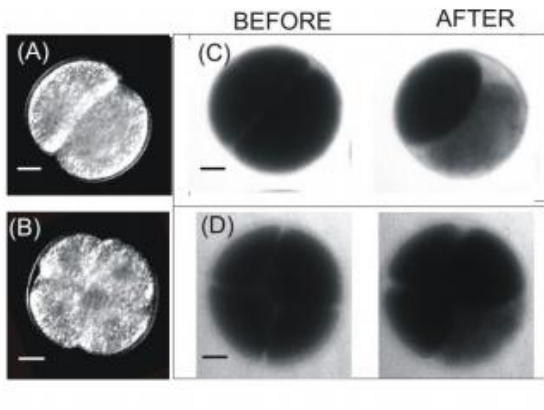

b)

FIGURE 13. Integral Imaging. a) Method, and b) image reconstruction (Torres-Mapa, et al., 2004)

At figure 13, we can see the method the integral imaging and their specification. Also, we can tell the difference of Integral Imaging. 


\section{Stereoscopic Display}

Past and present implementations of most 3DTV systems rely on stereoscopy, or multiview video. In these approaches, no attempt is made to duplicate the original optical field. Instead, two or more 2-D images are captured at slightly diff erent viewing angles. The human visual system interprets the received images. 3-D perception relies on the processing of several depth cues. Older type systems require special goggles to direct diff erent images to each eye; however, newer systems utilize autostereoscopic systems to guide diff erent 2-D views to diff erent angles (Sexton \& Surman, 1999).

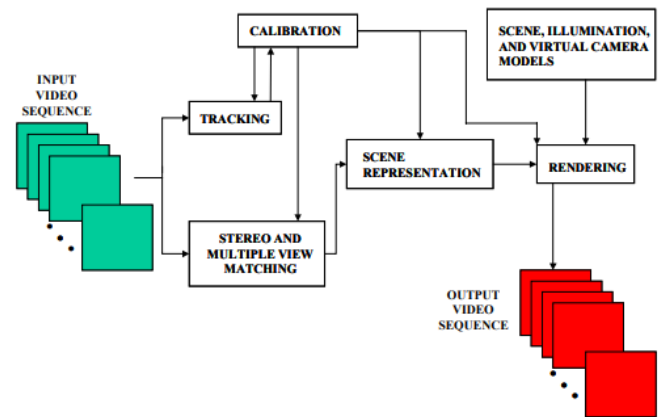

FIGURE 14. Stereoscopic Display on (Isgrò, et al., 2004)

Systems based on stereoscopic principles usually create a feeling like motion sickness especially when some associated alignments are imperfect. Signal processing issues related with such display schemes (Isgrò, et al., 2004). While the stereoscopy-based techniques are the most popular 3-D imaging techniques to date, holography-based techniques will most likely be the ultimate choice for digital holographic in the future.

\section{SUMMARY}

Holography was being technology of optics that records and reconstructs wave-fronts of light and have potential development for use in next- generation imaging technology with various applications such as optical data storage and three- dimensional holographic imaging. These fundamentals related to wave principle such as FFT, Propagational Wave, also Diffraction and Interference. Holography was divided to two classification, its $\mathrm{OH}$ and $\mathrm{DH}$.

\section{ACKNOWLEDGMENTS}

Thanks for Dr. Iwan Sugihartono, M.Si that giving us time to learn about Holography. Author declared that the article must be revised to be clear before going to publishing to the public. Any comment can help author to revise the article. Also, because so much literature in Holography, we recommended to read another literature in reference sect.

\section{REFERENCES}

Acharya, I. \& Upadhyay, D., 2015. Comparative Study of Digital Holography Reconstruction Methods. Procedia Computer Science, Volume 58, pp. 649-658. 
Ackermann, K. \& Eichler, J., 2007. Holography: A Practical Approach. 1st ed. New Jersey: John Wiley \& Sons.

Amako, J., Miura, H. \& Sonehara, T., 1993. Wavefront Control Using Liquid Crystal Devices. Applied Optics, 32(23), pp. 4323-4329.

Andrelevicius, M., 2011. Methods and Applications of Optical Holography. Material Science (MEDŽIAGOTYRA), 17(4), pp. 371-377.

B J, J. \& P K, P., 2013. Fast computational method for digital Cylindrical holography, Chennai: Anna University.

Beev, K. et al., 2006. Total Internal Reflection Holographic Gratings Recorded in Polymerdispersed Liquid Crystals. Optics Communications, Volume 260, pp. 192-195.

Benton, S., 1977. White Light Transmission / Reflection Holographic. s.1., The Pergamon press, pp. 401409.

Born, M. \& Wolf, E., 1999. Principles of Optics. London, Cambridge University Press.

Gabor, D., 1948. A New Microscopic Principle. Nature, 161(1), pp. 777-778.

Gabor, D., 1951. Microscopy by Reconstructed Wavefronts. London, Royal Society ,pp .449 -469.

Geng, J., 2013. Three- dimensional display technologies. Adv. Opt. Photon, 5(4), pp. 456 - 535.

Hariharan, P., 1996. Optical Holography: Principles, Techniques, and Application. London: Cambridge University Press.

Hariharan, P., 2002. Basics of Holography. 2nd ed. London: Cambridge University Press.

Isgrò, F., Trucco, E., Kauff, P. \& Schreer, O., 2004. 3-D Image Processing in The Future. s.1., IEEE Transactions on Circuits and Systems for Video Technology.

Jeong, T., 1967. Cylindrical holography and some proposed applications. J.Opt.Soc.Am, 57(1), pp. 13961398.

Jesacher, A. et al., 2008. Near-Perfect Hologram Reconstruction with a Spatial Light Modulator. Optics Express, 16(4), p. 2600.

Johnston, S. F., 2006. Holographic Visions: A History of New Science. 1st ed. Oxford: Oxford University Press.

Kim, M. K., 2011. Principle of Holography. In: Digital Holographic Microscopy. 1st ed. New York: Springer Link, pp. 29 - 37.

Lohman, A. \& Paris, D., 1967. Binary Fraunhofer Holograms Generated by Computer. Appl.Opt., Volume 6, pp. 1739 - 1748. 
Okano, F., Hoshino, H., Arai, J. \& Yayuma, I., 1997. Real Time Pickup Method for a Three-Dimensional Image Based on Integral Photography. Applied Optics, Volume 36, pp. 1598-1603.

Ozcan, M. \& Bayraktar, M., 2009. Digital Holography Image Reconstruction Methods. s.1., SPIE.

Pain, H., 2005. The Physics of Vibrations and Waves. 6th ed. London: John Wiley \& Sons Ltd.

Poon, T.-C., 2006. Digital Holography and Three-Dimensional Display: Principles and Applications. 1st ed. New York: Springer.

Saleh, B. \& Teich, M., 1991. Fundamentals of Photonics. New York: Wiley.

Sexton, I. \& Surman, P., 1999. Stereoscopic and Autostereoscopic Display Systems. IEEE Signal Processing Magazine, 16(3), pp. 85-99.

Torres-Mapa, M. L. et al., 2004. Integrated Holographic System for All-Optical Manipulation of developing embryos. Biomedical Optics Express, 2(6), pp. 1566-1569.

Trolinger, J., 1975. Particle Field Holography. Optical Engineering, Volume 14, pp. 383-392.

Vander, L. A., 1964. Signal detection by complex spatial filtering. Sydney, IEEE Transactions on Information Theory, IT-10. 